Peter Wingren, Mathematics Department, UmeåUniversity, S-901 87 Umeå, Sweden. e-mail: Peter.Wingren@math.umu.se

\title{
DIMENSIONS OF GRAPHS OF FUNCTIONS AND LACUNARY DECOMPOSITIONS OF SPLINE APPROXIMATIONS
}

\begin{abstract}
A construction is put forward which shows how to decompose an arbitrary continuous function $f \in C[0,1]$ into a sum of two continuous functions each having a graph of Hausdorff dimension one. An example is given where the terms in the decomposition have graphs of Hausdorff dimension 1 and packing dimension 2 .
\end{abstract}

\section{Introduction}

In [4] Mauldin and Williams proved, by an easy Baire category argument that it is possible to decompose an arbitrary function $f(x) \in C[0,1]$ into a sum of two functions $s(x) \in C[0,1]$ and $t(x) \in C[0,1]$ such that the Hausdorff dimensions of the graphs of $s$ and $t$ are one. This decomposition is surprising since there are functions in $C[0,1]$ which have graphs of any dimension in the interval $[1,2]$.

We believe that it is of interest to see how the decomposition can be constructed. From our construction one sees that the decomposition is far from unique and that it is possible to generalize it to $C\left(\mathbb{R}^{n}\right)$.

One can expect that a constructive proof gives more information than an existence proof. Indeed, it is possible to carry out the construction so that the decomposition functions $s$ and $t$ have graphs of lower box dimension 1 . This is done in Theorem 1. Moreover it is possible to make a detailed investigation of the decomposition functions $s=s_{f}$ and $t=t_{f}$ for special cases of $f$. This is done in Theorem 2 and it gives an answer to an interesting question posed by

Key Words: decomposition, continuous function, graph of continuous function, Haussdorff dimension, packing dimension

Mathematical Reviews subject classification: 28A12, 28A78 and 28A80

Received by the editors April 12, 1996 
an anonymous referee concerning the packing dimension of the decomposition functions. We prove that there are functions $f$ such that

$$
\operatorname{dim}_{P}\left(s_{f}\right)=\operatorname{dim}_{P}\left(t_{f}\right)=2
$$

and hence the irregularity coefficient [6]

$$
\operatorname{dim}_{P}(\cdot)-\operatorname{dim}_{H}(\cdot)
$$

attains the maximum value 1 if the arguments are $s_{f}$ and $t_{f}$ respectively. Noteworthy is that this value is as large as the maximum value for arbitrary real valued functions defined on $[0,1]$.

Before we begin with the proofs we give a rough outline of the construction. The first step is to approximate $f(x)$ by the functions in a uniformly convergent sequence $\left\{f_{n}\right\}$ of linear spline functions of increasing frequency and accuracy. A telescopic series is then formed from the sequence and the terms in the series are sorted in two sums $s$ and $t$ with partial sums $s_{i}$ and $t_{i}$ so that

1. $f=s_{i}+t_{i}+f-f_{n_{i}} i=1,2, \ldots$ and

2. $s_{i}$ and $t_{i}$ converge uniformly to $s$ and $t$ on $[0,1]$.

From this it follows that

$$
f=s+t
$$

where $s$ and $t$ are continuous. We now arrive at the heart of the construction. The pair $(s, t)$ in the decomposition $s+t$ may be varied extensively. We use this to choose a very high convergence speed for $\left\{s_{i}\right\}$ and $\left\{t_{i}\right\}$ and we distribute the oscillation for $f$ so unevenly between $s$ and $t$ that $\operatorname{graph}(s)$ and $\operatorname{graph}(t)$ inherit (almost linear) covering properties from graph $\left(s_{i}\right)$ and graph $\left(t_{i}\right)$ for long sequences of scales. This will imply that $s$ and $t$ inherit the property of having graphs of lower box dimension 1 and hence of Hausdorff dimension 1 from the graphs of the spline functions $s_{i}$ and $t_{i}$.

In Theorem 2 we prove, for a special function $f$ and by a detailed investigation of the corresponding functions $s$ and $t$, that their graphs have upper box dimension 2 and since the graphs are very homogenous it is possible to prove that their packing dimensions are 2 .

We now leave this rough description and prepare for theorems and proofs by recalling some definitions. In the following, we use the squares $I_{n, k} \times I_{n, j}$ where $I_{n, k}=\left[k \cdot 2^{-n},(k+1) 2^{-n}\right], n \in \mathbb{Z}_{+}$and $k, j \in \mathbb{Z}$. We call these squares dyadic 2 -cubes. It is well known that the $s$-dimensional net measure defined by dyadic 2 -cubes defines the same dimension as the $s$-dimensional Hausdorff measure (see e.g. [3, p. 33]). We will use the net measure, as in our construction, it 
is a little easier to handle. Define for $F \subset \mathbb{R}^{2}$ the $s$-dimensional net measure $M^{s}(F)$ by

$$
M^{s}(F):=\lim _{n \rightarrow \infty} \inf _{2^{-n}} \sum_{i}\left|Q_{i}\right|^{s}
$$

where the $Q_{i}^{\prime} s$ are dyadic 2-cubes which cover $F$ and which are of side length less than or equal to $2^{-n}$. The infimum is taken over all coverings of such cubes. Roughly speaking the $s$-dimensional net measure is a measure of how effective $F$ can be covered by dyadic 2-cubes in the $x^{s}$-sense.

In the proof, we call $\sum_{i}\left|Q_{i}\right|^{s}$ the covering sum. The Hausdorff dimension of $F$ is defined by

$$
\operatorname{dim}_{H}(F):=\inf \left\{s: M^{s}(F) \text { is finite }\right\}
$$

If every dyadic cube in a covering sum has the same side length $2^{-n}$, then

$$
\underline{S}(s)=\liminf _{n \rightarrow \infty} \sum_{i}\left|Q_{i}\right|^{s} \text { and } \bar{S}(s)=\limsup _{n \rightarrow \infty} \sum_{i}\left|Q_{i}\right|^{s}
$$

may be used to define the lower- and the upper box dimension given by

$$
\underline{\operatorname{dim}}_{B}=\inf \{s: \underline{S}(s) \text { is finite }\} \text { and } \overline{\operatorname{dim}}_{B}=\inf \{s: \bar{S}(s) \text { is finite }\}
$$

respectively. If the reader would like to investigate how the constructions in the proofs work for various functions then we suggest trying those functions whose construction is based either upon dyadic decomposition, the Takagi function [5, p. 176] and the family of functions in $\left[1, \mathrm{p}\right.$. 873] with $\operatorname{dim}_{H}=1$, the Ursell-Besicovith functions [2, p. 115] with $\operatorname{dim}_{H}=\alpha, \alpha \in(1,2)$ or the function $\sum_{n=0}^{\infty} 2^{-n} \inf \left\{\left|2^{2^{n}} x-y\right|: y\right.$ integer $\}\left[7\right.$, p. 104] with $\operatorname{dim}_{H}=2$.

\section{Decomposition of an Arbitrary $C[0,1]$ Function}

Functions in this section are defined on the unit interval $[0,1]$. We sometimes exclude the independent variable, writing for instance $f$ instead of $f(x)$.

Theorem 1. Any real-valued continuous function $f$ on $[0,1]$ may be decomposed into a sum of two continuous functions $s$ and $t, f(x)=s(x)+t(x)$ where the graphs of $s$ and $t$ have lower box dimension 1 .

The corresponding result by Mauldin and Williams for the Hausdorff dimension follows from Theorem 1: 
Corollary 1. Any real-valued continuous function $f$ on $[0,1]$ may be decomposed into a sum of two continuous functions s and $t, f(x)=s(x)+t(x)$ where the graphs of $s$ and $t$ have Hausdorff dimension 1.

Proof. In the exposition below we use the dyadic intervals

$I_{n, k}=\left[k \cdot 2^{-n},(k+1) 2^{-n}\right], n \in Z_{+}$and $0 \leq k \leq 2^{-n}-1$, when constructing the two functions $s(x)$ and $t(x)$. We start by first letting $f_{n}, n=1,2, \ldots$, be the linear splines which interpolate $f(x)$ at $x=k \cdot 2^{-n}, k=0,1, \ldots, 2^{n}$. Since $f$ is continuous on $[0,1]$ we have

$$
\lim _{n \rightarrow \infty} \sup _{x \in[0,1]}\left|f_{n}(x)-f(x)\right|=0
$$

Then we let

$$
\begin{aligned}
g_{1} & =f_{1} \\
g_{2} & =f_{2}-f_{1} \\
& \vdots \\
g_{n} & =f_{n}-f_{n-1} .
\end{aligned}
$$

The function $f(x)=\sum_{1}^{\infty} g_{i}(x)$ has uniform convergence. To get $s$ and $t$ we decompose $f(x)=\sum_{1}^{\infty} g_{i}(x)$ into a sum $f=s+t$ where

$$
s=\sum_{\text {odd }} \sum_{k}^{N_{k+1}} g_{i} \text { and } t=\sum_{\text {even }} \sum_{k}^{N_{k+1}} g_{i}
$$

In these sums, $N_{1}, N_{2}, \ldots$ is an increasing sequence of natural numbers formed by a special inductive rule. We specify this rule and prove that

$$
\underline{\operatorname{dim}}_{B}(\operatorname{graph}(s))=\underline{\operatorname{dim}}_{B}(\operatorname{graph}(t))=1 .
$$

Step 1. Let $h_{1}=\sum_{N_{0}+1}^{N_{1}} g_{i}, \quad N_{0}=0$ and $N_{1}=1$. Cover graph $\left(h_{1}\right)$ by dyadic 2-cubes, all with side length $2^{-n}$. Choose $n=n_{1}$ large enough so that the number of dyadic 2-cubes which cover graph $\left(h_{1}\right)$ multiplied by the side-length $2^{-n_{1}}$ raised to the power of $\left(1+\frac{1}{p}\right), p=1$, is less than 1 . Thus

$$
\#\left\{2 \text { - cubes which cover graph }\left(h_{1}\right)\right\} \cdot\left(2^{-n_{1}}\right)^{1+\frac{1}{1}}<1 .
$$


This choice is possible since $h_{1}$ is a linear spline and hence rectifiable.

Step 2. Let

$$
h_{2}=\sum_{N_{1}+1}^{N_{2}} g_{i}
$$

where $N_{2}$ is so large that $\left|\sum_{n}^{\infty} g_{i}\right|<2^{-n_{1}}$ for $n>N_{2}$. The choice of $N_{2}$ is possible since $\sum g_{i}(x)$ is uniformly convergent on $[0,1]$.

Let us now assume that we have already made the choices in steps $1,2,3, \ldots, p-1$ and that $p$ is odd; if $p$ is even the wording in the proof is the same except for some minor details. We then have

$$
\begin{aligned}
h_{1} & =\sum_{N_{0}+1}^{N_{1}} g_{i} \\
h_{2} & =\sum_{N_{1}+1}^{N_{2}} g_{i} \\
& \vdots \\
h_{p-1} & =\sum_{N_{p-2}+1}^{N_{p-1}} g_{i}
\end{aligned}
$$

with integers $N_{1}<N_{2}<\ldots<N_{p-1}$ and $n_{1}<n_{2}<\ldots<n_{p-1}$.

Step $p$. Let $h_{p}=\sum_{N_{p-1}+1}^{N_{p}} g_{i}$ where $N_{p}>N_{p-1}$ is sufficiently large that

$$
\left|\sum_{n}^{\infty} g_{i}\right|<2^{-n_{p-1}}, n>N_{p}
$$

Now cover graph $\left(h_{1}+h_{3}+\ldots+h_{p}\right)$ with 2 -cubes of side-length $2^{-n_{p}}$ where we choose $n_{p}$ large enough so that $n_{p}>n_{p-1}$ and so that

$$
\#\left\{2 \text {-cubes which cover graph }\left(h_{1}+h_{3} \ldots+h_{p}\right)\right\} \times\left(2^{-n_{p}}\right)^{1+\frac{1}{p}}<1 \text {. }
$$

This choice is justified in the same way as the choice of $n_{1}$ in step 1 ; just replace $h_{1}$ by $h_{1}+h_{3}+\ldots+h_{p}$. By induction it follows that we can continue with the construction so that (6) and (5) hold for every positive integer $p$. Our next goal is to prove that for a given $\varepsilon>0$ there is a $p_{0}>0$ such that it is 
possible to cover the graph of $s=\sum_{i=0}^{\infty} h_{2 i+1}$ by dyadic 2-cubes of side lengths $2^{-n_{p}}$, so that

$$
\text { \#\{covering } 2 \text {-cubes }\} \times\left(2^{-n_{p}}\right)^{1+\varepsilon}<1+\varepsilon \text {. }
$$

for every $p \geq p_{0}>0$. From this it follows by (1) and (2) that

$$
\underline{\operatorname{dim}}_{B}(\operatorname{graph}(s)) \leq 1
$$

and since $s$ is continuous we have $1 \leq \underline{\operatorname{dim}}_{B}(\operatorname{graph}(s))$. Thus $\underline{\operatorname{dim}}_{B}(\operatorname{graph}(s))=$ 1. To prove (7) we observe that from (4), (5) and the inductive construction, it follows that for $k=1,2, \ldots$

$$
\begin{aligned}
\left|h_{k}\right| & =\left|\sum_{N_{k-1}+1}^{N_{k}} g_{i}\right|=\left|\sum_{N_{k-1}+1}^{\infty} g_{i}-\sum_{N_{k}+1}^{\infty} g_{i}\right| \\
& \leq\left|\sum_{N_{k-1}+1}^{\infty} g_{i}\right|+\left|\sum_{N_{k}+1}^{\infty} g_{i}\right| \leq 2^{-n_{k-1}}+2^{-n_{k}} \leq 2 \cdot 2^{-n_{k-1}},
\end{aligned}
$$

and hence

$$
\left|\sum_{\text {even } i \geq m} h_{i}\right| \leq \sum_{\text {even } i \geq m}\left|h_{i}\right| \leq 4 \cdot 2^{-n_{m-1}}
$$

From (6) it follows that there is an $n_{p}$ for the above given $\varepsilon>0$ for which $\operatorname{graph}\left(\sum_{\text {even } i \leq p} h_{i}\right)$ is covered by 2 -cubes of side length $2^{-n_{p}}$ so effectively that

$$
\#\left\{\text { covering } 2 \text {-cubes of sidelength } 2^{-n_{p}}\right\} \times\left(2^{-n_{p}}\right)^{1+\varepsilon}<1 \text { for } p \geq p_{0} .
$$

But according to (8) $\left|\sum_{\text {even } i \geq p+2} h_{i}\right|<4 \cdot 2^{-n_{p}}$. Hence it is possible to cover $\operatorname{graph}\left(\sum_{\text {even } i} h_{i}\right)$ by adding to the covering of $\operatorname{graph}\left(\sum_{\text {even } i \leq p} h_{i}\right) 4$ levels of 2-cubes of side length $2^{-n_{p}}$ from the above and 4 levels from below; that is $8 \times\left(1 / 2^{-n_{p}}\right)$ cubes. This number of 2 -cubes adds $8 \cdot 2^{n_{p}} \cdot\left(2^{-n_{p}}\right)^{1+\varepsilon}$ to the covering sum. Since this quantity approaches zero as $p$ tends to infinity, there is a $p_{0}$ for the cover of $\operatorname{graph}(s)$ so that

$$
\#\left\{\text { covering } 2 \text {-cubes of side length } 2^{-n_{p}}\right\} \times\left(2^{-n_{p}}\right)^{1+\varepsilon}<1+\varepsilon
$$

if $p \geq p_{0}$ and the proof is complete. 


\section{Decomposition of a Special $C[0,1]$ Function}

Given the decomposition functions $s=s_{f}$ and $t=t_{f}$ as in Theorem 1 we have the following theorem.

Theorem 2. There are continuous functions $f(x), x \in[0,1]$, such that

$$
\operatorname{dim}_{P}(F)=2
$$

and

$$
\operatorname{dim}_{P}(F)-\operatorname{dim}_{H}(F)=1
$$

for $F \in\left\{\operatorname{graph}\left(s_{f}\right), \operatorname{graph}\left(t_{f}\right)\right\}$.

Proof. It is obvious that (9) implies (10) for the decomposition functions we have in Theorem 1. Furthermore, if there is one $f$ which fulfils (9) then there are several.

We prove (9) for

$$
f(x)=\sum_{p=0}^{\infty} 2^{-p} \operatorname{dist}\left(2^{2^{p}} x, \mathbb{Z}\right), x \in[0,1] .
$$

This function was given in [7] as an example of a continuous function whose graph is of Hausdorff dimension 2. Our reasoning relies on Theorem 1 and its proof and consists of three parts which we first sketch and later fill in the details.

1. Prove $\overline{\operatorname{dim}}_{B}(t)=2$.

To attain this result, we start with a comparison between the local oscillation of the linked functions $f, s$, and $t$.

2. Show that graph $(t)$ has a high degree of homogeneity in the following sense: if we subtract from $t$ the linear spline function $\sigma_{r}$ in (15) below, then we get a periodic function. If $r \rightarrow \infty$ then the period approach zero.

3. Use the homogeneity property of $t$ to exploit a well known result (see for instance [3, p. 49]) concerning sufficient conditions for equality between the upper box dimension and the packing dimension: Let $F$ be a closed subset of $\mathbb{R}^{n}$. If for every open subset $V$ of $\mathbb{R}^{n}, V \cap F \neq \emptyset$, we have that

$$
\overline{\operatorname{dim}}_{B}(F \cap V)=\overline{\operatorname{dim}}_{B}(F)
$$

then $\operatorname{dim}_{P}(F)=\overline{\operatorname{dim}}_{B}(F)$.

Part 1. Let $f$ be the function in (11) and apply the decomposition in Theorem 1. Then

$$
f=s+t .
$$


We now introduce the following notation. If $I$ is an interval in $\mathbb{R}$ then

$$
|I|:=\operatorname{length}(I)
$$

and if $I$ is a dyadic interval then $\operatorname{cov}(f(I), I)$ stands for the number of dyadic intervals of length $|I|$ which intersect $f(I)$. Hence $|f(I)|$ is the oscillation of $f$ on $I$ and $\operatorname{cov}(f(I), I)$ is the number of dyadic 2-cubes of side length $|I|$ intersecting $\operatorname{graph}(f(x)), x \epsilon I$. From (13) it follows that $|f(I)| \leq|s(I)|+|t(I)|$ and

$$
\operatorname{cov}(f(I), I) \leq \operatorname{cov}(s(I), I)+\operatorname{cov}(t(I), I)+1 .
$$

Summation over all dyadic intervals $I$ of equal length gives

$$
\sum \operatorname{cov}(f(I), I) \leq \sum \operatorname{cov}(s(I), I)+\sum \operatorname{cov}(t(I), I)+|I|^{-1} .
$$

To enable us to draw conclusions about the upper box dimension of $t$, we let $\varepsilon>0$ and multiply the above inequality by $|I|^{2-\varepsilon}$. Then we get

$$
\begin{aligned}
\sum \operatorname{cov}(f(I), I)|I|^{2-\varepsilon} \leq & \sum \operatorname{cov}(s(I), I)|I|^{2-\varepsilon} \\
& +\sum \operatorname{cov}(t(I), I)|I|^{2-\varepsilon}+|I|^{1-\varepsilon} .
\end{aligned}
$$

Since $\operatorname{dim}_{H}(\operatorname{graph}(f))=2$, then replacing $|I|$ by $2^{-n}$ we know that the left hand side of the above inequality approaches infinity as $n \rightarrow \infty$. This is also true for any subsequence of $\mathbb{N}$ and specifically the subsequence $\left\{n_{p}\right\}$ which was defined in Theorem 1. We know however from (7) that the first term on the right hand side is less than $1+\varepsilon$ for every $n_{p}$. Hence the second term on the right hand side approach infinity as $p \rightarrow \infty$. This yields

$$
\overline{\operatorname{dim}}_{B}(f) \geq 2-\varepsilon, \text { for every } \varepsilon .
$$

Thus

$$
\overline{\operatorname{dim}}_{B}(\operatorname{graph}(t))=2 .
$$

Part 2. We begin by taking a closer look on $t$. From Theorem 1 and its proof we have

$$
t(x)=\sum_{m=1}^{\infty} \sum_{N_{m}+1}^{N_{m+1}} 2^{-p} \operatorname{dist}\left(2^{2^{p}} x, \mathbb{Z}\right)
$$

Introducing the notation

$$
\sigma_{r}(x)=\sum_{m=1}^{r} \sum_{N_{m}+1}^{N_{m+1}} 2^{-p} \operatorname{dist}\left(2^{2^{p}} x, \mathbb{Z}\right)
$$


we get

$$
t(x)-\sigma_{r}(x)=\sum_{m=r+1}^{\infty} \sum_{N_{m}+1}^{N_{m+1}} 2^{-p} \operatorname{dist}\left(2^{2^{p}} x, \mathbb{Z}\right)
$$

Since

$$
\operatorname{dist}\left(2^{2^{p}}\left(x+2^{-2^{n}}\right), \mathbb{Z}\right)=\operatorname{dist}\left(2^{2^{p}} x, \mathbb{Z}\right) \text { for all } n \leq p
$$

it follows that the right hand side of (16) is of period $2^{-2^{p}}$. Then with the exception of a finite number of points the corresponding graph is the union of $2^{2^{p}}$ disjoint subsets all congruent to $\left(i 2^{2^{p}},(i+1) 2^{2^{p}}\right) \times \mathbb{R} \cap \operatorname{graph}(t)$. If we use this together with the relation (14) and

$$
\overline{\operatorname{dim}}_{B}\left(\operatorname{graph}(t+\sigma)=\overline{\operatorname{dim}}_{B}(\operatorname{graph}(t)),\right.
$$

which is true for all splines $\sigma$ defined on $\mathbb{R}$, then we obtain

$$
\begin{aligned}
2 & =\overline{\operatorname{dim}}_{B}(\operatorname{graph}(t))=\overline{\operatorname{dim}}_{B}\left(\operatorname{graph}\left(t-\sigma_{r}\right)\right) \\
& =\overline{\operatorname{dim}}_{B}\left(\operatorname{graph}\left(t-\sigma_{r}\right) \cap\left[i 2^{2^{p}},(i+1) 2^{2^{p}}\right] \times \mathbb{R}\right) \\
& =\overline{\operatorname{dim}}_{B}\left(\operatorname{graph}(t) \cap\left[i 2^{2^{p}},(i+1) 2^{2^{p}}\right] \times \mathbb{R}\right)
\end{aligned}
$$

Part 3. In this last part we use the homogeneity property from Part 2 to prove (9). Assume that $V$ is an arbitrary open subset of $\mathbb{R}^{2}$ with $V \cap \operatorname{graph}(t) \neq$ $\emptyset$. Then since $V$ is open and $t$ is continuous there is an $i$ such that

$$
\operatorname{graph}(t) \cap V \supset \operatorname{graph}(t) \cap\left(\left[i 2^{2^{p}},(i+1) 2^{2^{p}}\right] \times \mathbb{R}\right)
$$

and since $\overline{\operatorname{dim}}_{B}$ is an increasing set function we achieve

$$
\begin{aligned}
2 & \geq \overline{\operatorname{dim}}_{B}(\operatorname{graph}(t)) \geq \overline{\operatorname{dim}}_{B}(\operatorname{graph}(t) \cap V) \\
& \geq \overline{\operatorname{dim}}_{B}\left(\operatorname{graph}(t) \cap\left(\left[i 2^{2^{p}},(i+1) 2^{2^{p}}\right]\right) \times \mathbb{R}\right) .
\end{aligned}
$$

We know however from (17) that the last expression in the above inequality equals 2 and hence

$$
\overline{\operatorname{dim}}_{B}(\operatorname{graph}(t) \cap V)=\overline{\operatorname{dim}}_{B}(\operatorname{graph}(t)) .
$$

Thus (12) is valid for $F=\operatorname{graph}(t)$ and hence by (14) we may conclude that (9) holds and with this the proof is complete.

Acknowledgement. The author thanks the referees for valuable suggestions. 


\section{References}

[1] A. Deliu and P. Wingren, The Takagi operator, Bernoulli sequences, smoothness conditions and fractal curves, Proc. Amer. Math. Soc. 121 (1994), 871-881.

[2] K. J. Falconer, The geometry of fractal sets, Cambridge Tracts in Math.,vol. 85 Cambridge Univ. Press, Cambridge and New York, 1985.

[3] K. J. Falconer, Fractal geometry, Mathematical Foundations and Applications, Wiley, New York, 1990.

[4] R. D. Mauldin and S .C. Williams, On the Hausdorff dimension of some graphs, Trans. Amer. Math. Soc. 298 (1986), 793-803.

[5] T. Takagi, A simple example of a continuous function without derivative, Proc. Phys. Math. Soc. Japan 1 (1903), 176-177.

[6] C. Tricot, Two definitions of fractional dimension, Math. Proc. Camb. Phil. Soc.91(1982), 57-74.

[7] P. Wingren, Concerning a real-valued continuous function on the interval with graph of Hausdorff dimension 2, L'enseignement Mathématique $4 \mathbf{1}$ (1995), 1103-110. 